\title{
Effect of Surface Electrical Stimulation in a Seated Position on Pelvic Floor Muscle Functions, Urinary Leakage, and Quality of Life in Women With Stress Urinary Incontinence
}

\section{Ui-jae Hwang}

Yonsei University - Maeji Campus: Yonsei University - Wonju Campus

Min-seok Lee

Sophie-Marceau Women's Clinic

Sung-hoon Jung

Yonsei University - Maeji Campus: Yonsei University - Wonju Campus

Young-shin Kim

Shera Women's clinic

Oh-yun Kwon ( $\square$ kwonoy@yonsei.ac.kr)

Yonsei University - Wonju Campus https://orcid.org/0000-0002-9699-768X

\section{Research article}

Keywords: Pelvic floor muscle, stress urinary incontinence, surface electrical stimulation

Posted Date: December 8th, 2020

DOI: https://doi.org/10.21203/rs.3.rs-120216/v1

License: (c) (1) This work is licensed under a Creative Commons Attribution 4.0 International License.

Read Full License 


\section{Abstract}

\section{Background}

The aim of this study was to demonstrate the effect of surface electrical stimulation in a seated position (SESSP), as pelvic floor muscle (PFM) training, on PFM functions (time to reach maximal pressure [TRMP], muscle strength, power and endurance), urinary leakage and quality of life in patients with stress urinary incontinence (SUI).

\section{Methods}

Women with SUI were randomized into an SESSP group $(n=18)$ or control group $(n=18)$. Quality of life were assessed by the Incontinence Quality of Life Questionnaire (I-QOL). Ultra-short pad test results and PFM functions were measured by perineometer. Changes in the outcomes were assessed before and 8 weeks after SESSP training.

\section{Results}

Thirty-three participants were included in the analysis. There were significant differences in I-QOL (avoidance and limiting behaviors, psychosocial impacts, social embarrassment and total score) in between (SESSP vs. control group) and within (pre vs. post) group analyses. Significant increases in PFM strength, power, and endurance, and significant decreases in the TRMP and pad weight, were observed between (SESSP vs. control group) and within (pre vs. post) groups.

\section{Conclusion}

SESSP in a seated position can be recommended to improve QOL, urinary leakage, and PFM functions in SUl patients.

Trial registration

Current Controlled Trials KCT0003357 (the date of registration: 2018.11.16) and retrospectively registered

\section{Background}

Stress urinary incontinence (SUI) is the involuntary loss of urine on exertion, effort, sports activities, coughing, or sneezing, as defined by the International Continence Society (ICS) [1]. The ICS reports that half of female urinary incontinence cases are attributable to SUI [1]. More than $30 \%$ of women develop SUI within 5 years after their first vaginal delivery [2]. Although SUI impacts quality of life (QOL), leading not only to physical limitations but also to psychosocial problems, patients often underreport SUI [3]. For mild to moderate SUI, all guidelines recommend noninvasive or conservative treatment before invasive treatment, because this can lead to better outcomes [3]. Noninvasive treatments include physical and 
behavioral therapy, such as bladder or pelvic floor muscle training (PFMT), electrical stimulation (ES), and vaginal cones.

The goal of noninvasive treatment for SUI is to enhance pelvic floor muscle (PFM) functions by increasing speed, power, strength, and endurance [4]. PFMT is a guideline-recommended primary treatment for SUI that aims to increase urethral pressure and tone, as well as PFM functions, and to decrease urethral hypermobility [2], thus preventing loss of urine and promoting continence [2]. However, it is difficult to initiate and train selective contraction of the PFM, particularly in incontinence patients who have difficulty with contracting the PFM selectively unassisted [5]. Also, the literature has shown that inappropriate PFM contractions can exacerbate the symptoms of SUI [5].

ES for PFMT could improve QOL, urinary leakage, and the pressure of selective PFM contractions. ES for PFMT has an improvement rate of $74 \%$, and a cure rate of $30-60 \%$, in patients with SUI [6]. Previous studies have reported the effects of ES as a treatment for SUI performed with superficial, anal, and intravaginal electrodes $[4,7,8]$. ES via transvaginal electrodes is frequently used in clinical practice, but the use of transvaginal electrodes could potentially be limited by pain, intolerance of the high stimulation intensity, discomfort associated with the insertion of electrodes, difficulty of hygienic management, and/or bleeding $[9,10]$.

Previous studies reported the effects of surface electrical stimulation in the supine or hook-lying position for treating urinary incontinence, which can be performed with placement of cutaneous electrodes in the perivaginal or sacral region $[7,11,12]$. Because PFM contraction in sitting or upright position was more difficult than hook-lying and supine position by vaginal squeeze pressure data [13], symptoms related to SUI could be occur mostly in sitting and standing. Also, difference of PFM strength according to position could reflect that it is necessary to training in a position where it is difficult to PFM contract $[13,14]$. However, to date, little has been reported on the effectiveness of ES for PFMT performed in a seated position using cutaneous electrodes implanted into both perivaginal and sacral regions for the treatment of SUI [15]. Additionally, there has been little study showing specific improvements of PFM functions (reaction time, muscle strength, power, and endurance) after applying surface electrical stimulation in a seated position (SESSP). Therefore, it is necessary to investigate the effectiveness of SESSP techniques for improving urinary incontinence and QOL.

The purpose of the present study was to determine the effectiveness of SESSP as PFMT performed in a seated position, as indexed by PFM functions (time to reach maximal pressure [TRMP], muscle strength, power and endurance), urinary leakage, and QOL. We hypothesized that PFM functions and QOL would improve, and urinary leakage would decrease, after 8 weeks of SESSP as PFMT in the experimental group compared with the control group.

\section{Materials And Methods}

\section{Subjects and design}


This was a randomized controlled trial (RCT) with an investigator-blinded parallel randomization (1:1) design (control and SESSP groups). The present study was performed at an obstetrics and gynecology clinic in Seoul, Korea, from August 2018 to November 2018. The sample size was determined a priori (version 3.1.3; University of Trier, Trier, Germany) in a pilot study with three participants in each group (experimental and control groups). The sample size was calculated according to a power of 0.80 , alpha level of 0.05 and effect size $f$ of 0.917 . The analysis indicated that more than six subjects were required. The women were recruited via advertisements included provided telephone contact information for the study. After the initial contact, visits were scheduled to review the participant suitability according to the inclusion and exclusion criteria of the study, and incontinence severity was checked via the IngelmanSundberg scale, as administered during an interview [16].

Inclusion criteria [15] were 1) SUI diagnosed by a urogynecologist, 2) a leakage episode occurring more than once a week, 3) generally healthy (apart from SUI), 4) body mass index $<30 \mathrm{~kg} / \mathrm{m}^{2}, 5$ ) age between 30 and 60 years, 6) non-smoker, 7) not addicted to alcohol or drugs, and 8) successfully completed medical screening questionnaire. Exclusion criteria [15] were 1) urogenital prolapse grade III or higher, 2) cardiac pacemakers, 3) devices implanted in the pelvis or hip joint, 4) pregnancy/planning to get pregnant, 5) pelvic or abdominal surgery within the last 6 months, 6) cognitive impairment, 7) concomitant treatment for SUI during the trial period, 8) neurological or psychiatric disease, and 9) urinary tract infection.

A total of 36 participants who met the inclusion criteria were allocated to control and SESSP groups through a list of random numbers generated by online randomization software (www.randomization.com) (Figure 1). Before the study, all procedures were explained to the participants, who then signed informed consent forms approved by the Institutional Review Board of Yonsei University Mirae Campus (1041849-201806-BM-056-02).

\section{Surface electrical stimulation device}

An SESSP device (EasyK7, Alphamedic Co., Ltd., Daegu, Korea) with three cutaneous electrodes placed in perivaginal (two electrodes) and sacral regions (one electrode) was used to stimulate the PFM and surrounding structures. Three cutaneous electrodes were inserted into the bottom and back of the SESSP device. This allowed for stimulation, via direct contact, of both the perivaginal and sacral regions, and created an electromagnetic field that stimulated the PFM while the participant sat on the SSEP (Figure 2). The initial stimulation amplitude was set by the physical therapist to determine the most comfortable level for each participant. The SSEP delivered biphasic, asymmetric impulses of $25 \mathrm{~Hz}$, and controlled the pulse (11 s) and rest (11 s) durations. The mean intensity among all participants was $19.13 \pm 5.47 \mathrm{~mA}$ (range: 2.5 to $30 \mathrm{~mA}$ ). Each SSEP session was 15 minutes in duration.

\section{Intervention}

Participants in the SESSP group were provided with an SSEP device, underwent their first SSEP session on site, and were educated on proper device usage, management, and cleaning. Individuals who 
experienced the SESSP sensation to be aversive were excluded from the study. Participants were instructed to use the device once a day (15-min session) 5 days a week for 8 weeks. All participants participated an SSEP session designed to determine the maximum stimulation amplitude that they could tolerate.

The Control group was asked to perform walking for 10 min and restrict PFMT regarding the PFM or abdominal muscle contraction. We gave and educated the SSEP to control group as a reward for participating in the experiment after 8 weeks. Both groups were evaluated at pre- and post- 8 weeks with repeated QOL questionnaires, as well as with ultra-short perineal pad tests and measurements of PFM functions performed using a perineometer.

\section{Outcomes}

The outcome of QOL was determined by patient self-assessment. Testers were blinded to the participant's responses to the questionnaires. The I-QOL evaluated incontinence-specific QOL [17]. The I-QOL consists of three health-related QOL factors: avoidance and limiting behaviors (eight items), psychosocial impact (nine items), and social embarrassment (five items); the Korean version was used in this study [18]. The IQOL has 22 items, each scored using a five-point scale. Three subscale scores and a total score are calculated. Scores for the 22 items were summed and then adjusted according to a 0-100 scale, with higher scores indicating higher QOL [17].

The objective status of incontinence was indicated by the ultra-short perineal pad test results and PFM power, strength, endurance, and TRMP was measured by perineometer. The ultra-short perineal pad test [19] was performed to assess urinary leakage status, as follows. The participants were allowed to empty their bladder, and any remaining urine was removed using a catheter without anesthetic gel. Then, the bladder was filled with $300 \mathrm{~mL}$ sterile water by a gynecologist using a catheter. For the pad test, the participants were provided with a pre-weighed sanitary diaper to place inside their underwear. Participants were instructed to perform a standardized physical activity for 1 minute at a submaximal level: jumping with feet apart and then together, jogging in place with high knees, and jumping up and down; 20 repetitions of each exercise and additional jogging in place were performed until 1 minute had passed [19]. After 10 minutes, the total amount of urine leakage was determined by weighing the pad.

The assessments of PFM functions were performed in a hook-lying position for all participants using a vaginal pressure measurement device. The VVP-3000 perineometer (QLMED Ltd., Gyeonggi-do, Korea), which is a vaginal probe $24 \mathrm{~mm}$ in diameter and $115 \mathrm{~mm}$ in length with an inserted surface measurement length of $66 \mathrm{~mm}$, was used. The vaginal probe is connected to a microprocessor with latex tubing, allowing for transmission of pressure readings when the inserted part is compressed by external pressure. The baseline pressure value was recorded in $\mathrm{mmHg}$ with no voluntary PFM contraction, and the device was then zeroed. Participants were asked to contract their PFM and squeeze with maximum effort for 2-3 seconds. They were instructed to pull their PFM in and up as much as possible, and with no use of extra-pelvic muscle contraction. The measurement was performed before the first contraction and was displayed as a flat curve, after the participants was asked to relax and to slowly breathe in and out [20]. 
PFM strength was given by the difference between the resting and peak pressure measurement, and reported as the mean of two maximal pressures (in $\mathrm{mmHg}$ ) [20]. TRMP was measured from the onset of contraction until maximal pressure was reached, and recorded in seconds. Measurement of muscle power required the participants to perform the contraction at maximal pressure as fast as possible. The present study defined PFM power as PFM strength/TRMP $(\mathrm{mmHg} / \mathrm{s})$. The endurance is the ability of a muscle to maintain sub-maximal or maximal force, assessed as the time over which a participant is able to sustain a maximal isometric contraction [20]. PFM endurance was measured as the mean contraction pressure for $10 \mathrm{~s}$, during one attempt $(\mathrm{mmHg})$.

\section{Statistical analysis}

All statistical analyses were conducted using SPSS software (ver. 18.0; SPSS Inc., Chicago, IL, USA). A $p$ value of 0.05 was used to indicate statistical significance. Kolmogorov-Smirnov Z-tests were used to verify that the data were normally distributed. Analysis of covariance (ANCOVA) was used to compare the groups before and after the intervention, with the baseline values used as covariates. Data are presented as mean \pm standard deviation. The effect sizes and confidence interval $(\mathrm{Cl})$ for the primary outcomes were calculated to determine the clinical significance of the data. An effect size $(r)$ is constrained between 0 (no correlation) and 1 (perfect correlation); in this study, $0 \leq r<0.1$ was classified as no effect, $0.1 \leq r<$ 0.3 as a small effect, $0.3 \leq r<0.5$ as a moderate effect, and $r \geq 0.5$ as a large effect.

\section{Results}

Thirty-six participants were randomly divided between the SESSP and control groups $(n=18$ participants per group). However, two participants in the SESSP group and one in the control group dropped out, citing a lack of time. Therefore, 33 participants completed the interventions and were included in the analysis (Table 1 and Figure 1).

Table 2 shows the outcomes, respectively, before and after the intervention (Table 2). There were significant differences in the $\mathrm{I}-\mathrm{QOL}$ avoidance and limiting behaviors parameter between the groups (mean change: $-12.798,95 \% \mathrm{Cl}:-17.887$ to -7.709 , effect size: $0.468, p<0.001$ ), and in psychosocial impacts (mean change: $-13.463,95 \% \mathrm{Cl}:-19.734$ to -7.193 , effect size: $0.391, \mathrm{p}<0.001$ ), social embarrassment (mean change: $-15.129,95 \% \mathrm{Cl}:-22.964$ to -7.294 , effect size: $0.341, p<0.001$ ) and total score (mean change: $-10.990,95 \% \mathrm{Cl}:-15.759$ to -6.220 , effect size: $0.425, \mathrm{p}<0.001$ ).

A significant difference was observed between groups in pad weight (mean change: $4.466,95 \%$ Cl: 2.298 to 6.634, effect size: $0.371, p<0.001$ ). Regarding the PFM functions, there were significant differences between groups in TRMP (mean change: $0.190,95 \% \mathrm{Cl}$ : 0.022 to 0.357 , effect size: $0.151, \mathrm{p}=0.028$ ), strength (mean change: $-2.973,95 \% \mathrm{Cl}:-5.605$ to -0.341 , effect size: $0.151, \mathrm{p}=0.028$ ), power (mean change: $-8.583,95 \% \mathrm{Cl}:-13.111$ to -4.055 , effect size: $0.333, p=0.001$ ), and endurance (mean change: $-3.508,95 \% \mathrm{Cl}:-6.006$ to -1.009 , effect size: $0.215, p=0.008)$. 


\section{Discussion}

Previous studies have demonstrated the benefits of PFMT for SUI [7]. Conventional ES devices for PFMT generally use a vaginal or anal electrode, which is invasive and often causes discomfort and injury and leads to low compliance. This can largely be attributed to the fact that the size of the vaginal space limits electrode size and the ability of the user to reach an intensity sufficient to elicit PFMT, given the relatively high current density [15]. Thus, the evidence supporting the use of PFMT employing ES for SUI has been equivocal $[10,15]$. After 8 weeks of SESSP for PFMT, the present study observed improvement in PFM functions, urinary leakage and QOL. SESSP for PFMT over 8 weeks was effective for treating SUI, demonstrating that this is a good option for improving PFM functions, urinary leakage and QOL.

Regarding PFM strength measured by perineometer to assess PFM contraction pressure, a previous study also found an increase in pressure following SESSP [7]. The present study evaluated various PFM functions based on perineometer data. Muscle strength was defined by Sale as the peak force during a maximal voluntary contraction, while muscle power was defined as the rate at which peak force is performed under a given time [21]. Muscle endurance is the ability of a muscle to maintain near maximal, and is given by the time over which a subject could sustain a maximal isometric contraction [20]. After 8 weeks of SESSP training in the present study, TRMP was significantly decreased, and PFM strength, power, and endurance were significantly increased. Also, there were significant differences in PFM functions between factors (SESSP group vs. control group).

The increases in PFM functions after SESSP training can be explained as follows. First, it may have been due to stimulating the pudendal nerve by SESSP $[22,23]$. Because surface electrode in sacral region could stimulate pudendal nerve, thus strengthening its muscle fibers, improving electric activation, and increasing proprioception and coordination during pelvic floor contraction [23]. Second, electrode pad in sacral region could restrict pelvic posterior tilt with sacral nutation movement. Sacral counter-nutation movement could more contract PFM compared to sacral nutation movement [24]. Thus, restriction of pelvic posterior tilting might more contract PFM during SESSP. Third, PFM contraction on ES could cause perturbations in myofibers and the extracellular matrix in the case of stimulus overload of the PFM [25]. In addition, the order of motor unit recruitment (from smallest to largest, according to Henneman's size principle) is controversial in ES-evoked muscle contractions; a more reasonable explanation is the random pattern of recruitment of fast- and slow-twitch muscles [25]. PFM is composed of $70 \%$ type I slow-twitch fibers and $30 \%$ type II fast-twitch fibers. Thus, ES-evoked contractions could stimulate the PFM according to the random pattern of recruitment of fast- and slow-twitch muscles, and enhance PFM strength and power by increasing the recruitment of fast-twitch muscles, and endurance by increasing the recruitment of slow-twitch muscle. Also, a previous study reported that significantly decreased force occurred after 30 seconds of high-frequency $(75 \mathrm{~Hz})$ EMS [26]. During this period of high-frequency force fatigue, increased force occurred at a low frequency $(20 \mathrm{~Hz})[26]$.

Previous studies have found improvements in urinary leakage, as evaluated by 1 -h pad tests in women treated with SESSP, which is consistent with the results of the present study $[7,8]$. Another study reported 
similar improvements in 20-min pad tests [4]. In the present study, pad weight decreased significantly after 8 weeks of SESSP training and there was a significant difference between the SESSP and control groups.

Currently, QOL is considered to be more relevant than quantitative measurements, because it reflects the participants' satisfaction with the treatment. This study found significant improvements in avoidance and limiting behaviors, psychosocial impact, social embarrassment, and total I-QOL score, both within (pre vs. post) and between (SESSP group vs. control group) groups. Two previous studies also reported improvements in QOL after SESSP, although this was assessed using the King's Health Questionnaire [7, 8].

The decrease in urinary leakage during pad tests and increase in I-QOL after 8 weeks of SESSP training may have been due to increasing PFM functions affecting the proprioception and coordination of pelvic floor contractions during situations that cause SUI [7]. During impact activities, such as running, jumping, and dancing, the PFM power requirement for SUI patients is highest, to decrease urinary leakage [27]. Thus, with changes in PFM power, the mechanism of urethral closure and SUI improves during ultra-short pad tests and impact activities [7,22]. Moreover, the pudendal nerve is an efferent nerve of the external urethral sphincter, so this treatment can increase the pressure of urethral closure, thus improving SUI [7].

The main limitation of this study was the absence of an electromyographic assessment to measure changes in PFM activity. Another limitation was that we did not utilize ultrasonography to evaluate whether PFM hypertrophy occurs after ES treatment.

\section{Conclusion}

SESSP could be considered for SUI patients, to improve QOL, urinary leakage, and PFM functions. SESSP opens up new possibilities for additional studies, which should include a follow-up session, urodynamic examination, and evaluation of changes in PFM thickness and volume, and in muscle activity (assessed using sonography and electromyography), to confirm the efficacy of SESSP.

\section{Abbreviations}




\begin{tabular}{ll} 
Cl & Confidence interval \\
\hline ES & Electrical stimulation \\
\hline I-QOL & Incontinence Quality of Life Questionnaire \\
\hline PFM & Pelvic floor muscle \\
\hline PFMT & Pelvic floor muscle training \\
\hline QOL & Quality of life \\
\hline SESSP & Surface electrical stimulation in a seated position \\
\hline SUI & Stress urinary incontinence \\
\hline TRMP & Time to reach maximal pressure
\end{tabular}

\section{Declarations}

\section{Ethics approval and consent to participate}

The present study was approved by the Institutional Review Board of Yonsei University Mirae Campus, Republic of Korea (1041849-201806-BM-056-02). The entire protocol including its rationale and objective, safety consideration and the participants'role was explained to each candidate in Korean. The participants were then asked to sign a written informed consent. The informed consent was made in two identical copies that the participants could retain one.

\section{Consent to publish}

In present study, using consent for publication was obtained from participants to publish potentially identifying details. The participants gave written to sign a informed consent for personal or clinical details along with any identifying images to be published in this study..

\section{Availability of data and materials}

The datasets used and/or analyzed during the present study are available from the corresponding author on reasonable request.

\section{Competing Interests}

The authors declare that they have no potential conflicts of interest with respect to the research, authorship, and publication of this article. The results are presented clearly, honestly, and without fabrication, falsification, or inappropriate data manipulation.

\section{Funding}


Yonsei University Research Fund (grant numbers: 2018-51-0213 and 2020-52-0016) provided funding for this study: SSEP devices provided free of charge, costs related to clinic visits/lab charges. No direct payment was made to individual study investigators. Funder about present study was OYK.

\section{Author contribution}

UJH, OYK made contributions to conception, design and analysis of the study and revised the manuscript. SHJ drafted the manuscript and performed the statistical analysis. MSL participated in the design of the study and acquisition of data. All authors read and approved the final manuscript.

\section{Acknowledgments}

We would like to thank all of the participants for their time and commitment to the present study.

\section{References}

1. Abrams P, Andersson K-E, Birder L, Brubaker L, Cardozo L, Chapple C, Cottenden A, Davila W, De Ridder D, Dmochowski R: Fourth International Consultation on Incontinence Recommendations of the International Scientific Committee: Evaluation and treatment of urinary incontinence, pelvic organ prolapse, and fecal incontinence. Neurourology and Urodynamics: Official Journal of the International Continence Society 2010, 29(1):213-240.

2. Bø K: Pelvic floor muscle training is effective in treatment of female stress urinary incontinence, but how does it work? International Urogynecology Journal 2004, 15(2):76-84.

3. Townsend MK, Minassian VA, Okereke OI, Resnick NM, Grodstein F: Urinary incontinence and prevalence of high depressive symptoms in older black versus white women. International urogynecology journal 2014, 25(6):823-829.

4. Castro RA, Arruda RM, Zanetti MR, Santos PD, Sartori MG, Girão MJ: Single-blind, randomized, controlled trial of pelvic floor muscle training, electrical stimulation, vaginal cones, and no active treatment in the management of stress urinary incontinence. Clinics 2008, 63(4):465-472.

5. Bø K, Mørkved S, Frawley H, Sherburn M: Evidence for benefit of transversus abdominis training alone or in combination with pelvic floor muscle training to treat female urinary incontinence: a systematic review. Neurourology and Urodynamics: Official Journal of the International Continence Society 2009, 28(5):368-373.

6. Shamliyan TA, Kane RL, Wyman J, Wilt TJ: Systematic review: randomized, controlled trials of nonsurgical treatments for urinary incontinence in women. Annals of internal medicine 2008, 148(6):459-473.

7. Correia GN, Pereira VS, Hirakawa HS, Driusso P: Effects of surface and intravaginal electrical stimulation in the treatment of women with stress urinary incontinence: randomized controlled trial. European Journal of Obstetrics \& Gynecology and Reproductive Biology 2014, 173:113-118. 
8. Pereira V, Bonioti L, Correia G, Driusso P: Effects of surface electrical stimulation in older women with stress urinary incontinence: a randomized controlled pilot study. Actas Urológicas Españolas (English Edition) 2012, 36(8):491-496.

9. Bø K, Talseth T, Holme I: Single blind, randomised controlled trial of pelvic floor exercises, electrical stimulation, vaginal cones, and no treatment in management of genuine stress incontinence in women. Bmj 1999, 318(7182):487-493.

10. Sand PK, Richardson DA, Staskin DR, Swift SE, Appel RA, Whitmore KE, Ostergard DR: Pelvic floor electrical stimulation in the treatment of genuine stress incontinence: a multicenter, placebocontrolled trial. American journal of obstetrics and gynecology 1995, 173(1):72-79.

11. Krauss DJ, Lilien O: Transcutaneous electrical nerve stimulator for stress incontinence. The Journal of urology 1981, 125(6):790-793.

12. Fall M: Electrical pelvic floor stimulation for the control of detrusor instability. Neurourology and Urodynamics 1985, 4(4):329-335.

13. Frawley HC, Galea MP, Phillips BA, Sherburn M, Bø K: Effect of test position on pelvic floor muscle assessment. International Urogynecology Journal 2006, 17(4):365-371.

14. $B \varnothing \mathrm{K}$, Finckenhagen $\mathrm{HB}$ : Is there any difference in measurement of pelvic floor muscle strength in supine and standing position? Acta obstetricia et gynecologica Scandinavica 2003, 82(12):11201124.

15. Maher RM, Caulfield B: A Novel Externally Applied Neuromuscular Stimulator for the Treatment of Stress Urinary Incontinence in Women-A Pilot Study. Neuromodulation: Technology at the Neural Interface 2013, 16(6):590-594.

16. Ingelman-Sundberg A, Ulmsten U: Surgical treatment of female urinary stress incontinence. In: Female stress incontinence. Volume 10, edn.: Karger Publishers; 1983: 51-69.

17. Wagner T, Patrick DL, Bavendam T, Martin M, Buesching D: Quality of life of persons with urinary incontinence: development of a new measure. Urology 1996, 47(1):67-71.

18. Park J, Shin DW, You C, Chung KJ, Han DH, Joshi HB, Park HK: Cross-cultural application of the Korean version of Ureteral Stent Symptoms Questionnaire. Journal of endourology 2012, 26(11):1518-1522.

19. Persson J, Bergqvist CE, Wølner-Hanssen P: An ultra-short perineal pad-test for evaluation of female stress urinary incontinence treatment. Neurourology and Urodynamics: Official Journal of the International Continence Society 2001, 20(3):277-285.

20. Tennfjord MK, Engh ME, Bø K: An intra-and interrater reliability and agreement study of vaginal resting pressure, pelvic floor muscle strength, and muscular endurance using a manometer. International urogynecology journal 2017, 28(10):1507-1514.

21. Sale D, Norman R: Testing strength and power. Physiological testing of the high-performance athlete 1991:21-106.

22. Spruijt J, Vierhout M, Verstraeten R, Janssens J, Burger C: Vaginal electrical stimulation of the pelvic floor: a randomized feasibility study in urinary incontinent elderly women. Acta obstetricia et 
gynecologica Scandinavica 2003, 82(11):1043-1048.

23. Terlikowski R, Dobrzycka B, Kinalski M, Kuryliszyn-Moskal A, Terlikowski SJ: Transvaginal electrical stimulation with surface-EMG biofeedback in managing stress urinary incontinence in women of premenopausal age: a double-blind, placebo-controlled, randomized clinical trial. International urogynecology journal 2013, 24(10):1631-1638.

24. Pool-Goudzwaard A, van Dijke GH, van Gurp M, Mulder P, Snijders C, Stoeckart R: Contribution of pelvic floor muscles to stiffness of the pelvic ring. Clinical Biomechanics 2004, 19(6):564-571.

25. Gregory CM, Bickel CS: Recruitment patterns in human skeletal muscle during electrical stimulation. Physical therapy 2005, 85(4):358-364.

26. Moritani T, Muro M, Kijima A: Electromechanical changes during electrically induced and maximal voluntary contractions: electrophysiologic responses of different muscle fiber types during stimulated contractions. Experimental neurology 1985, 88(3):471-483.

27. Hung $\mathrm{H}-\mathrm{C}$, Hsiao S-M, Chih S-Y, Lin H-H, Tsauo J-Y: An alternative intervention for urinary incontinence: retraining diaphragmatic, deep abdominal and pelvic floor muscle coordinated function. Manual Therapy 2010, 15(3):273-279.

\section{Tables}

Tables 1 and 2 are not available with this version

\section{Figures}

\section{Image not available with this version}

\section{Figure 1}

Flow diagram of our randomized trial in women with stress urinary incontinence 


\section{Image not available with this version}

Figure 1

Flow diagram of our randomized trial in women with stress urinary incontinence

\section{Image not available with this version}

Figure 2

Surface electrical stimulation in a seated position device (Alphamedic Co., Ltd.).

\section{Image not available with this version}

Figure 2

Surface electrical stimulation in a seated position device (Alphamedic Co., Ltd.). 


\section{Supplementary Files}

This is a list of supplementary files associated with this preprint. Click to download.

- CONSORT2010ChecklistMSWord.doc

- CONSORT2010ChecklistMSWord.doc 